\title{
Medium-Frequency Oscillation in Voltage-Controlled Full-Bridge Inverter with a Nonlinear Inductor
}

\author{
Faqiang Wang ${ }^{1, *} \mathbb{0}$, Hongwei Zhou ${ }^{1,2}$, Jiansong Zhang ${ }^{1,2}$ and Hongbo Cao ${ }^{1}$ \\ 1 State Key Laboratory of Electrical Insulation and Power Equipment, School of Electrical Engineering, \\ Xi'an Jiaotong University, Xi'an 710049, China; zhouhongwei@stu.xjtu.edu.cn (H.Z.); \\ zjs2013@stu.xjtu.edu.cn (J.Z.); caohongbo@stu.xjtu.edu.cn (H.C.) \\ 2 TBEA Xi'an Electric Technology Co., Ltd., Xi'an 710119, China \\ * Correspondence: faqwang@mail.xjtu.edu.cn; Tel.: +29-82668630-218
}

Citation: Wang, F.; Zhou, H.; Zhang, J.; Cao, H. Medium-Frequency Oscillation in Voltage-Controlled Full-Bridge Inverter with a Nonlinear Inductor. Energies 2021, 14, 3848. https://doi.org/10.3390/en14133848

Academic Editor: Alon Kuperman

Received: 26 April 2021

Accepted: 18 June 2021

Published: 25 June 2021

Publisher's Note: MDPI stays neutral with regard to jurisdictional claims in published maps and institutional affiliations.

Copyright: (c) 2021 by the authors. Licensee MDPI, Basel, Switzerland. This article is an open access article distributed under the terms and conditions of the Creative Commons Attribution (CC BY) license (https:/ / creativecommons.org/licenses/by/ $4.0 /)$.

\begin{abstract}
An inverter, whose inductor current is periodic, is the key equipment for photovoltaic power generation, fuel cell power generation, etc. A nonlinear inductor, whose inductance will be changed by its current, can be used to improve the grid current quality of the inverter, although making the mathematical model of the inverter system more complex. In this paper, the voltagecontrolled full-bridge inverter with a nonlinear inductor is investigated and its mathematical model is established. The influence of parameters on the dynamical behaviors of this system is analyzed by using the harmonic balance method and the Floquet theory. Consequently, the mechanism for the occurrence of medium-frequency oscillation is revealed. The hardware circuit for the voltagecontrolled full-bridge inverter with a nonlinear inductor is designed and some experimental results are presented for confirmation. The research results show that the mechanism for the occurrence of medium-frequency oscillation is Hopf bifurcation and its oscillation frequency is between the line frequency and the switching frequency.
\end{abstract}

Keywords: full-bridge inverter; mathematical model; medium-frequency oscillation; Hopf bifurcation

\section{Introduction}

Switching power converters, which include DC-DC converters, power factor correction (PFC) converters, DC-AC inverters, etc., mainly consist of semiconductors, inductors, capacitors, and loads, and have been widely employed in practical engineering systems such as photovoltaic (PV) power generation, fuel cell power generation, etc. [1]. For inductors in the switching power converters, as indicated in [2,3], two aspects need to be considered. (1) In order to reduce the THD of the system's current and improve the whole system's efficiency, the inductor needs to have a sufficiently high inductance under lightload current. (2) Under heavy-load current, it is hoped that the designed inductor would have low inductance to ensure that the inductor is in an unsaturated state. Obviously, it will be difficult for linear inductors to satisfy both these requirements simultaneously. In order to solve this problem, the nonlinear inductor has been proposed and designed [3-8]. For example, a ladder-gap nonlinear inductor whose inductance can be varied with the specified load was designed in [3]. Based on low-temperature co-fired ceramic (LTCC) technology, the multiwindow multipermeability nonlinear LTCC inductor was fabricated and applied to a DC-DC buck converter in [4]. In [5], the nonlinear inductor was employed in the interleaved power factor correction (PFC) converter, its Jiles-Atherton model was established, and the dynamic behaviors of the system were investigated. In [6], efficient nonlinear inductors were adopted in PV inverters and active PFC converters; the corresponding design method for nonlinear inductors was presented, where the research results showed its ability to perform at a size $32 \%$ smaller than linear inductors, with $20 \%$ less copper, and a total power loss that is $10 \%$ lower by using nonlinear inductors instead of linear inductors in a practical PV inverter. Furthermore, some researchers have applied 
nonlinear inductors to the three-phase grid-connected inverter [7], single-phase PV grid converters [8], DC-DC boost converters [9], and so on. Certainly, the research results above have greatly promoted the design of nonlinear inductors and their applications in switching power converters.

In addition, the extant research results in [10-18] show that the inverter is a type of strongly nonlinear system so that there are rich and complex nonlinear phenomena which will inevitably have an important influence on the performance of the inverter. For instance, in [10], bifurcation and chaos in the single-phase sinusoidal pulse width modulation (SPWM) full-bridge inverter with a linear inductor were analyzed by using the discrete model, and an adaptive carrier amplitude modulation control method was presented to control these discovered instabilities in order to ensure its stable operation. In [11], the exponential delay feedback control method used for controlling the chaotic phenomenon in the single-phase H-bridge photovoltaic inverter with a linear inductor was reported. In [12], the fast-scale instability and slow-scale instability in a single-phase parallel full-bridge inverter with a linear inductor was analyzed. Likewise, the complex nonlinear phenomena of the dual buck full-bridge inverter with a linear inductor were studied in [13]. The bifurcation phenomenon in parallel-connected voltage-source inverters with a linear inductor was analyzed by using the delay-feedback control method in [15]. The above research results provide good guidance for the parameter design of the inverter system in practical engineering to ensure its stable operation.

These achievements were all carried out on inverters with linear inductors; that is, the mathematical model of the inverter systems are piecewise linear models so that the analytical solution in each segment could be obtained, and the discrete model could be used to analyze such systems. However, with the introduction of the nonlinear inductor whose characteristics will make the dynamic behaviors of the inverter system more complicated, analyzing its strongly nonlinear system with the use of the discrete model will certainly prove to be even more difficult. Therefore, it is necessary to study the inverter with a nonlinear inductor that aims to provide a theoretical basis and design guidance for its engineering applications.

In this paper, the mathematical model of the voltage-controlled full-bridge inverter with a nonlinear inductor is established, the influence of system's parameters on its dynamic behaviors is analyzed, and the mechanism of medium frequency oscillation in the system is revealed. Additionally, a hardware circuit for the system is designed, and some experimental results used to verify the correctness of the theoretical analyses and the existence of the medium-frequency oscillation phenomenon are presented.

\section{Nonlinear Inductor's Model and Its Experimental Verification}

For the nonlinear inductor, its inductance can be described by its static inductance and dynamic inductance [3]. The mathematical model for the nonlinear inductor's static inductance, provided in the system-level power electronics simulation software and named Piecewise Linear Electrical Circuit Simulation (PLECS) developed by the Swiss company Plexim $\mathrm{GmbH}$, is given as [19]:

$$
L_{E}=\mathrm{A}_{1}+\mathrm{A}_{2} \frac{\arctan \left(\mathrm{A}_{3} i_{N}\right)}{i_{N}}
$$

where $A_{1}, A_{2}$, and $A_{3}$ are the parameters of the nonlinear inductor, arctan is the arctangent function, $i_{N}$ is the current through the nonlinear inductor, and $L_{E}$ is the static inductance of the nonlinear inductor.

Note that the expression for the magnetic flux linkage of the nonlinear inductor is $\varphi$; $=L_{E} i_{N}$, i.e., $\varphi=\mathrm{A}_{1} i_{N}+\mathrm{A}_{2} \arctan \left(\mathrm{A}_{3} i_{N}\right)$. Subsequently, the voltage across this nonlinear 
inductor is $v_{L N}=d \varphi / d t$, that is, the relationship between the current $i_{N}$ through this nonlinear inductor and its voltage $v_{L N}$ satisfies the following formula:

$$
v_{L N}=\frac{\mathrm{A}_{1}+\mathrm{A}_{2} \mathrm{~A}_{3}+\mathrm{A}_{1} \mathrm{~A}_{3}{ }^{2} i_{N}{ }^{2}}{1+\mathrm{A}_{3}{ }^{2} i_{N}{ }^{2}} \frac{d i_{N}}{d t}
$$

Additionally, based on the definition of dynamic inductance $L_{N}$, its expression is the following:

$$
L_{N}=\frac{d \varphi}{d i_{N}}=\frac{\mathrm{A}_{1}+\mathrm{A}_{2} \mathrm{~A}_{3}+\mathrm{A}_{1} \mathrm{~A}_{3}{ }^{2} i_{N}{ }^{2}}{1+\mathrm{A}_{3} i_{N} i_{N}^{2}}
$$

The test circuit consisting of an AC voltage source $v_{S}(t)$, linear resistor $R$, nonlinear inductor $L_{N}$, and linear inductor $L$ is shown in Figure $1 \mathrm{a}$, where $R=1 \Omega, L=110 \mu \mathrm{H}$, and $v_{S}(t)=18 \sin (2000 \pi t) \mathrm{V}$. Notably, $L_{N}$ is the dynamic inductance of the nonlinear inductor. According to Figure 1a, the experimental test data including the voltage across the linear inductor $v_{L}$, the voltage across the nonlinear inductor $v_{L N}$, and the voltage across the linear resistor $v_{R}$ can be obtained. Additionally, since the linear resistor is $R=1 \Omega$, the current $i_{N}$ through the nonlinear inductor can be represented by the voltage $v_{R}$. Thus, the dynamic inductance of the nonlinear inductor can be calculated as follows:

$$
L_{N}=L \frac{v_{L N}}{v_{L}}
$$

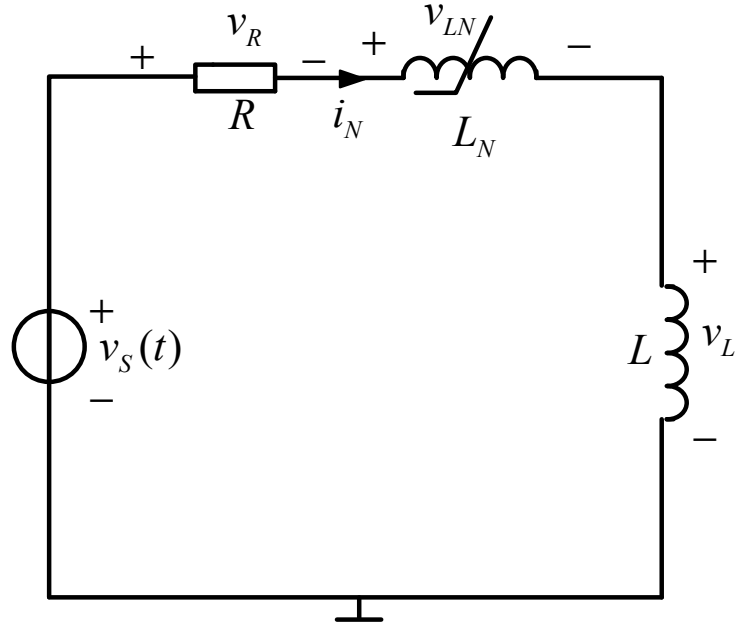

(a)

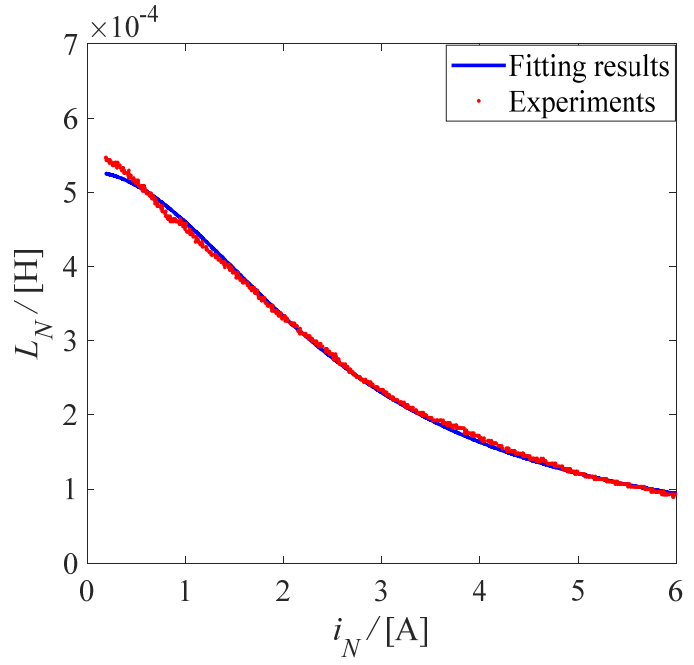

(b)

Figure 1. Test circuit of nonlinear inductor. (a) Circuit diagram, (b) Comparisons of nonlinear inductor fitting results and experiments.

By making the dynamic inductance $L_{N}$ described in (3) as the goal of the fitting function and substituting the test data obtained from the experiments in (3), $\mathrm{A}_{1}, \mathrm{~A}_{2}$, and $\mathrm{A}_{3}$ shown in (3) could be obtained by using the nlinfit function in the Matlab software; the fitting results are $\mathrm{A}_{1}=1.5845 \times 10^{-5}, \mathrm{~A}_{2}=0.0013$, and $\mathrm{A}_{3}=0.3932$. Figure $1 \mathrm{~b}$ shows the comparisons between the fitted nonlinear inductance $L_{N}$ with the current $i_{N}$ and the corresponding experimental test results. One can see that the results from these two sides are basically consistent with each other, indicating the effectiveness of the nonlinear inductor model and the correctness of the fitting technique.

\section{Mathematical Model and Approximate Calculation}

By replacing the linear inductor in the full-bridge inverter with the above-mentioned nonlinear inductor $L_{N}$, the circuit schematic diagram of the voltage-controlled full-bridge 
inverter with nonlinear inductor $L_{N}$ could be obtained and is shown in Figure 2. The power circuit of this system consists of an input $\mathrm{DC}$ voltage $v_{i n}$, four power switches $\left(\mathrm{Q}_{1}, \mathrm{Q}_{2}, \mathrm{Q}_{3}\right.$, and $\mathrm{Q}_{4}$ ), a nonlinear inductor $L_{N}$ (whose inductor current is $i_{N}$ ), a linear capacitor $C$ (whose voltage is $\left.v_{0}\right)$, and a load resistor $R_{L}$. Note that four power switches $\left(\mathrm{Q}_{1}, \mathrm{Q}_{2}, \mathrm{Q}_{3}\right.$, and $\left.\mathrm{Q}_{4}\right)$ form the inverter bridge, and they are turned on or off by their drive signals. Specifically, when the drive signal is at a high level, the corresponding power switches are turned on, otherwise they are turned off. Moreover, the driving signals of $Q_{1}$ and $Q_{4}$ are the same, the driving signals of $Q_{2}$ and $Q_{3}$ are the same, and $Q_{1}$ and $Q_{2}$ are turned on complementarily.

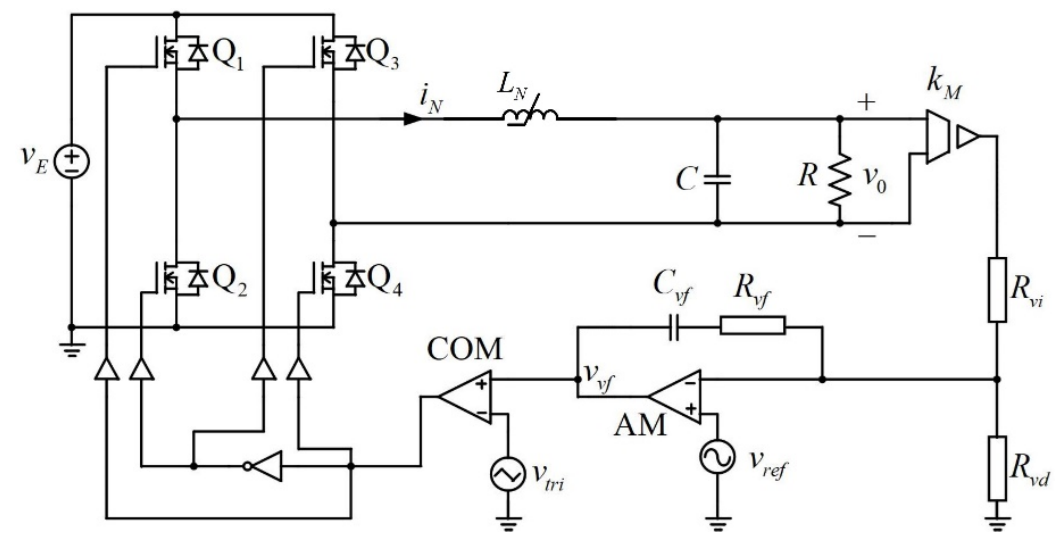

Figure 2. Schematic diagram of the voltage-controlled full-bridge inverter with nonlinear inductor.

The control circuit of this system is composed of the measurement circuit for the output floating voltage $v_{0}$, the bleeder circuit consisting of $R_{v i}$ and $R_{v d}$, the PI compensator, the SPWM generator, and the driving circuit. The output voltage of the PI compensator is $v_{v f}$ and $v_{r e f}=V_{\text {ref }} \sin \left(\omega_{0} t\right)$. Assuming that all components are ideal, i.e., their parasitic parameters are not considered since their values are very small, taking the voltage $v_{0}$, the current $i_{N}$, and the voltage $v_{v f}$ as the circuit variables, and based on the KCL and KVL law, the mathematical model of the system described by differential equations can be obtained as follows:

$$
\left\{\begin{array}{c}
\frac{d i_{N}}{d t}=\frac{1+\mathrm{A}_{3}{ }^{2} i_{N}{ }^{2}}{\mathrm{~A}_{1}+\mathrm{A}_{2} \mathrm{~A}_{3}+\mathrm{A}_{1} \mathrm{~A}_{3}{ }^{2} i_{N}{ }^{2}}\left((2 S-1) v_{E}-v_{0}\right) \\
\frac{d v_{0}}{d t}=\frac{i_{N}}{\mathrm{C}}-\frac{v_{0}}{R_{L} \mathrm{C}} \\
\frac{d v_{v f}}{d t}=\frac{k_{M} R_{v f} v_{0}}{R_{v i} C R_{L}}-\frac{k_{M} v_{0}}{R_{v i} C_{v f}}-\frac{k_{M} R_{v f} i_{N}}{R_{v i} \mathrm{C}}+k_{1} \sin \left(\omega_{0} t\right)+k_{2} \cos \left(\omega_{0} t\right)
\end{array}\right.
$$

where

$$
\left\{\begin{array}{c}
k_{1}=\left(\frac{1}{R_{v i}}+\frac{1}{R_{v d}}\right) \frac{V_{r e f}}{C_{v f}} \\
k_{2}=\left(\frac{R_{v f}}{R_{v i}}+\frac{R_{v f}}{R_{v d}}+1\right) V_{r e f} \omega_{0}
\end{array}\right.
$$

Notably, $S=1$ means that $\mathrm{Q}_{1}$ and $\mathrm{Q}_{4}$ are turned on, while $\mathrm{Q}_{2}$ and $\mathrm{Q}_{3}$ are turned off. $S$ $=0$ means that $Q_{2}$ and $Q_{3}$ are turned on, while $Q_{1}$ and $Q_{4}$ are turned off. Clearly different from the full-bridge inverter with a linear inductor, Equation (5) is always nonlinear for both $S=1$ and $S=0$ so that it will be difficult to obtain its analytical solution, and the discrete model cannot be adopted to analyze the stability of the voltage-controlled fullbridge inverter with a nonlinear inductor.

As for the bipolar SPWM generator, it consists of a comparator COM, a triangular carrier $v_{t r i}$, and a power switch driver. Note that the triangle carrier $v_{t r i}$ is expressed as follows:

$$
v_{t r i}=\left\{\begin{array}{l}
-\left(\omega_{s w} t-2 \pi k-2 \pi\right) \frac{2 V_{t r i}}{\pi}-V_{t r i}, 2 \pi k+\pi \leq \omega_{s w} t \leq 2 \pi k+2 \pi \\
\left(\omega_{s w} t-2 \pi k\right) \frac{2 V_{t r i}}{\pi}-V_{t r i}, 2 \pi k \leq \omega_{s w} t \leq 2 \pi k+\pi
\end{array}\right.
$$


where $\omega_{s w}$ and $V_{t r i}$ are the angular frequency and the amplitude of the triangular carrier, respectively. The triangle carrier $v_{t r i}$ is compared with the voltage $v_{v f}$ to generate the SPWM signal. If $v_{t r i}<v_{v f}$, the comparator COM output is at a high level, while if $v_{t r i}>v_{v f}$, the comparator COM outputs a low level.

For the full-bridge inverter with a nonlinear inductor, the angular frequency $\omega_{s w}$ of the triangular carrier $v_{t r i}$ is much larger than the angular frequency $\omega_{0}$ of the modulating wave $v_{r e f}$; that is, the switching frequency $f_{s w}\left(f_{s w}=\omega_{s w} / 2 \pi\right)$ is much larger than the power frequency $f_{0}\left(f_{0}=\omega_{0} / 2 \pi\right)$. Therefore, the average modeling method proposed in [20] can be used to establish the average model of the system, and the duty ratio $\langle d\rangle$ can be computed as follows:

$$
d=\frac{1}{2}\left(1+\frac{\left\langle v_{v f}\right\rangle}{V_{t r i}}\right)
$$

Therefore, the average model of the voltage-controlled full-bridge inverter with a nonlinear inductor can be given as:

$$
\left\{\begin{array}{l}
\frac{d\left\langle i_{N}\right\rangle}{d t}=\frac{1+\mathrm{A}_{3}{ }^{2}\left\langle i_{N}\right\rangle^{2}}{\mathrm{~A}_{1}+\mathrm{A}_{2} \mathrm{~A}_{3}+\mathrm{A}_{1} \mathrm{~A}_{3}{ }^{2}\left\langle i_{N}\right\rangle^{2}}\left(\frac{v_{E}}{V_{t r i}}\left\langle v_{v f}\right\rangle-\left\langle v_{0}\right\rangle\right) \\
\frac{d\left\langle v_{0}\right\rangle}{d t}=\frac{\left\langle i_{N}\right\rangle}{C}-\frac{\left\langle v_{0}\right\rangle}{R_{L} C} \\
\frac{d\left\langle v_{v f}\right\rangle}{d t}=\frac{k_{M} R_{v f}\left\langle v_{0}\right\rangle}{R_{v i} C R_{L}}-\frac{k_{M}\left\langle v_{0}\right\rangle}{R_{v i} C_{v f}}-\frac{k_{M} R_{v f}\left\langle i_{N}\right\rangle}{R_{v i} \mathrm{C}}+k_{1} \sin \left(\omega_{0} t\right)+k_{2} \cos \left(\omega_{0} t\right)
\end{array}\right.
$$

where $\langle d\rangle,\left\langle i_{N}\right\rangle,\left\langle v_{0}\right\rangle$, and $\left\langle v_{v f}\right\rangle$ are the average values of $d, i_{N}, v_{0}$, and $v_{v f}$ in one switching period, respectively.

One can see that the voltage-controlled full-bridge inverter with a nonlinear inductor described by (9) is a complex nonlinear and non-autonomous circuit system. Generally speaking, for this inverter system, it is expected that the average value of its output voltage $v_{0}$ should be approximately a sine wave, and the harmonic is expected to be as low as possible; therefore, it can be assumed as:

$$
\left\langle v_{0}\right\rangle=a_{0}+\sum_{n=1}^{N}\left[a_{n} \cos (n \tau)+b_{n} \sin (n \tau)\right]
$$

where $\tau=\omega_{0} t$ and

$$
\left\{\begin{array}{l}
\left\langle v_{0}^{\prime}\right\rangle=\frac{d\left\langle v_{0}\right\rangle}{d t} \\
\left\langle v^{\prime \prime} 0\right\rangle=\frac{d^{2}\left\langle v_{0}\right\rangle}{d t^{2}} \\
\left\langle v_{0}\right\rangle{ }^{(-1)}=\int\left\langle v_{0}\right\rangle d t
\end{array}\right.
$$

hence,

$$
\left\{\begin{array}{l}
\left\langle i_{N}\right\rangle=C\left\langle v \prime_{0}\right\rangle+\left\langle v_{0}\right\rangle / R_{L} \\
\left\langle v_{v f}\right\rangle=\frac{V_{t r i}}{v_{E}}\left(\left\langle i \prime_{N}\right\rangle \frac{\left(\mathrm{A}_{1}+\mathrm{A}_{2} \mathrm{~A}_{3}\right)+\mathrm{A}_{1} \mathrm{~A}_{3}{ }^{2}\left\langle i_{N}\right\rangle^{2}}{1+\mathrm{A}_{3}{ }^{2}\left\langle i_{N}\right\rangle^{2}}+\left\langle v_{0}\right\rangle\right) \\
\left\langle i \prime_{N}\right\rangle=C\left\langle v \prime_{0}\right\rangle+\left\langle v \prime_{0}\right\rangle / R_{L}
\end{array}\right.
$$

By substituting the first expression and the third expression of (12) with (9), we can get the following:

$$
\begin{aligned}
& f\left(a_{0}, a_{1}, \ldots, a_{N}, b_{1}, \ldots, b_{N}\right)=\left(\mathrm{A}_{1}+\mathrm{A}_{2} \mathrm{~A}_{3}+\mathrm{A}_{1} \mathrm{~A}_{3}{ }^{2}\left(C\left\langle v_{0}^{\prime}\right\rangle+\frac{\left\langle v_{0}\right\rangle}{R_{L}}\right)^{2}\right) \\
& \left(C\left\langle v^{\prime \prime}{ }_{0}\right\rangle+\frac{\left\langle v_{0}^{\prime}\right\rangle}{R_{L}}\right)-\left(1+\mathrm{A}_{3}{ }^{2}\left(C\left\langle v^{\prime}{ }_{0}\right\rangle+\frac{\left\langle v_{0}\right\rangle}{R_{L}}\right)^{2}\right)\left(\frac { v _ { E } } { V _ { t r i } } \left(\frac{k_{M} R_{v f}\left\langle v_{0}\right\rangle^{(-1)}}{R_{v i} C R_{L}}\right.\right. \\
& \left.\left.-\frac{k_{M}\left\langle v_{0}\right\rangle^{(-1)}}{R_{v i} C_{v f}}-\frac{k_{M} R_{v f}\left(C\left\langle v_{0}\right\rangle+\left\langle v_{0}\right\rangle^{(-1)} / R_{L}\right)}{R_{v i} C}-\frac{k_{1}}{\omega_{0}} \cos (\tau)+\frac{k_{2}}{\omega_{0}} \sin (\tau)\right)-\left\langle v_{0}\right\rangle\right)
\end{aligned}
$$

Notably, if the bigger number $N$ is selected, more accurate results can be obtained. However, by considering that the amplitudes of high order harmonics are very small when compared to that of the fundamental, choosing a small value for $N$ is enough. Then, 
substituting (10) and (11) with (13) and taking $N=4$, and based on the principle of harmonic balance, we can get the following:

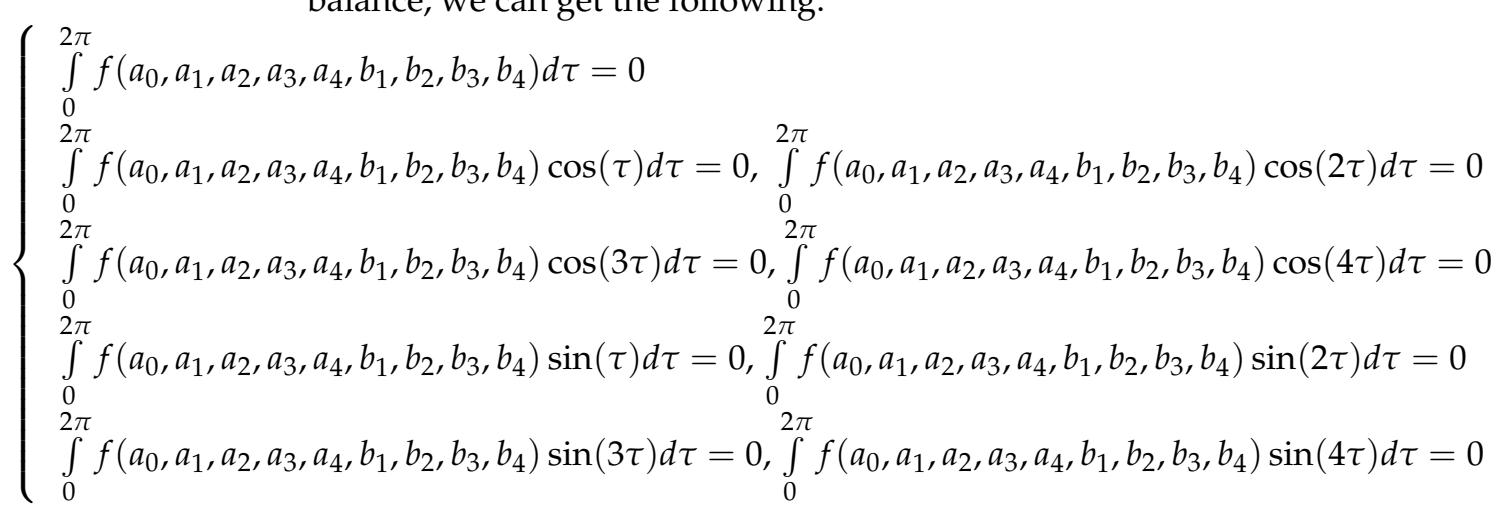

By solving (14), the coefficients $a_{0}, a_{1}, a_{2}, a_{3}, a_{4}, b_{1}, b_{2}, b_{3}$, and $b_{4}$ can be calculated by using the improved differential evolution method, and the expression for the average output voltage $\left\langle v_{0}\right\rangle$ of the voltage-controlled full-bridge inverter with a nonlinear inductor can be obtained.

The circuit parameters of the voltage-controlled full-bridge inverter with a nonlinear inductor are chosen as $v_{E}=12 \mathrm{~V}, C=20 \mu \mathrm{F}, R_{L}=5 \Omega, V_{\text {tri }}=2 \mathrm{~V}, V_{\text {ref }}=1 \mathrm{~V}, f_{\text {sw }}=25 \mathrm{kHz}$, $f_{0}=50 \mathrm{~Hz}, C_{v f}=102 \mathrm{nF}, R_{v f}=1 \mathrm{k} \Omega, R_{v i}=36 \mathrm{k} \Omega, R_{v d}=36 \mathrm{k} \Omega$, and $k_{M}=0.25$. The approximate solution of the voltage $\left\langle v_{0}\right\rangle$ is calculated as:

$$
\begin{aligned}
\left\langle v_{0}\right\rangle= & -0.187207-1.263136 \cos (\tau)+7.158382 \sin (\tau) \\
& -0.100880 \cos (2 \tau)-0.178769 \sin (2 \tau)-0.057748 \cos (3 \tau) \\
& -0.137595 \sin (3 \tau)-0.018889 \cos (4 \tau)-0.109892 \sin (4 \tau)
\end{aligned}
$$

Likewise, the current $\left\langle i_{N}\right\rangle$ and the voltage $\left\langle v_{v f}\right\rangle$ from (12) can be obtained.

Figure 3 shows the comparisons between the numerical simulations obtained from (9) and the approximate calculation results obtained from the harmonic balance (HB) method. It can be seen that the results from these two sides are consistent with each other, which indicates that the harmonic balance method is effective for calculating the approximate solution of the voltage-controlled full-bridge inverter with a nonlinear inductor.

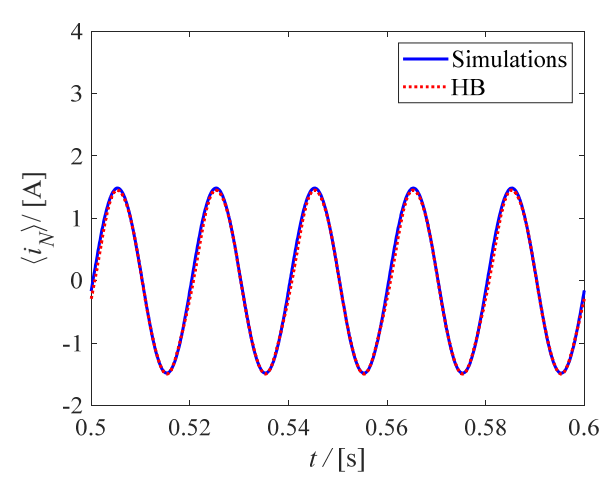

(a)

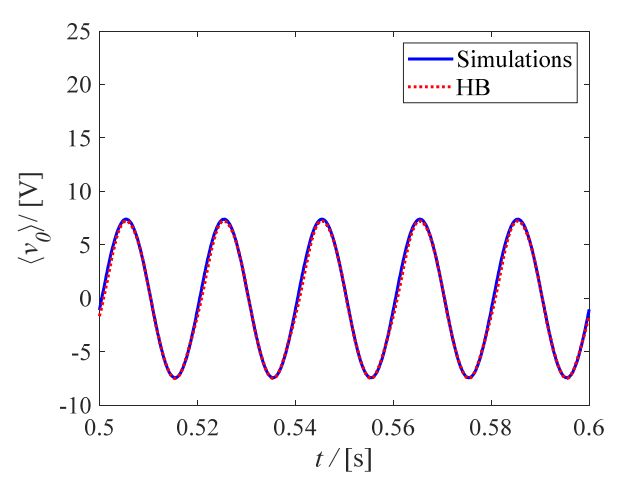

(b)

Figure 3. Comparison of numerical simulations and the results from HB. (a) $\left\langle i_{N}\right\rangle ;$ (b) $\left\langle v_{0}\right\rangle$.

\section{Theoretical Analysis on Oscillation Phenomena}

From (9), it can be seen that the voltage-controlled full-bridge inverter with a nonlinear inductor is a type of periodic time-varying nonlinear non-autonomous system, and its solution is the periodic solution as shown in (15). Therefore, the Floquet theory can be used 
to judge the stability of the periodic solution descried in (15). Supposing that this system has a small perturbation near its periodic solution, the following equation can be used:

$$
\left\{\begin{array}{l}
i_{N}=\left\langle i_{N}\right\rangle+\Delta i_{N} \\
v_{0}=\left\langle v_{0}\right\rangle+\Delta v_{0} \\
v_{v f}=\left\langle v_{v f}\right\rangle+\Delta v_{v f}
\end{array}\right.
$$

where

$$
\left\{\begin{array}{l}
\left\langle i_{N}\right\rangle \gg \Delta i_{N} \\
\left\langle v_{0}\right\rangle \gg \Delta v_{0} \\
\left\langle v_{v f}\right\rangle \gg \Delta v_{v f}
\end{array}\right.
$$

Then, linearize (9) at its periodic solution $\left(\left\langle i_{N}\right\rangle,\left\langle v_{0}\right\rangle,\left\langle v_{v f}\right\rangle\right)$, the linearization matrix $\mathbf{Y}(\tau)$ can be obtained as follows:

$$
\mathbf{Y}(\tau)=\left[\begin{array}{lll}
\frac{a_{11}}{\omega_{0}} & \frac{a_{12}}{\omega_{0}} & \frac{a_{13}}{\omega_{0}} \\
\frac{1}{\omega_{0} C} & -\frac{1}{\omega_{0} R_{L} C} & 0 \\
-\frac{k_{M} R_{v f}}{\omega_{0} R_{v i} C} & \frac{k_{M} R_{v f}}{\omega_{0} R_{v i} C R_{L}}-\frac{k_{M}}{\omega_{0} R_{v i} C_{v f}} & 0
\end{array}\right]
$$

where

$$
\left\{\begin{aligned}
a_{11} & =\left(\frac{v_{E}}{V_{\text {tri }}}\left\langle v_{v f}\right\rangle-\left\langle v_{0}\right\rangle\right)\left(\frac{2 \mathrm{~A}_{3}{ }^{2}\left\langle i_{N}\right\rangle}{\mathrm{A}_{1}+\mathrm{A}_{2} \mathrm{~A}_{3}+\mathrm{A}_{1} \mathrm{~A}_{3}{ }^{2}\left\langle i_{N}\right\rangle^{2}}-\frac{2 \mathrm{~A}_{1} \mathrm{~A}^{2}{ }^{2}\left\langle i_{N}\right\rangle\left(1+\mathrm{A}_{3}{ }^{2}\left\langle i_{N}\right\rangle^{2}\right)}{\left(\mathrm{A}_{1}+\mathrm{A}_{2} \mathrm{~A}_{3}+\mathrm{A}_{1} \mathrm{~A}_{3}{ }^{2}\left\langle i_{N}\right\rangle^{2}\right)^{2}}\right) \\
a_{12} & =-\frac{\left.1+\mathrm{A}_{3} i_{N}\right\rangle^{2}}{\mathrm{~A}_{1}+\mathrm{A}_{2} \mathrm{~A}_{3}+\mathrm{A}_{1} \mathrm{~A}_{3}{ }^{2}\left\langle i_{N}\right\rangle^{2}} \\
a_{13} & =\frac{1+\mathrm{A}_{3}{ }^{2}\left\langle i_{N}\right\rangle^{2}}{\mathrm{~A}_{1}+\mathrm{A}_{2} \mathrm{~A}_{3}+\mathrm{A}_{1} \mathrm{~A}_{3}{ }^{2}\left\langle i_{N}\right\rangle^{2}} \frac{v_{E}}{V_{\text {tri }}}
\end{aligned}\right.
$$

The period of the linearization matrix $\mathbf{Y}(\tau)$ is consistent with the period of $\left\langle v_{0}\right\rangle$. Therefore, the linearized system can be expressed as follows:

$$
\dot{\mathbf{x}}=\mathbf{Y}(\tau) x, \quad \mathbf{x}=\left(\Delta i_{N} ; \Delta v_{0} ; \Delta v_{v f}\right) \in R^{3}
$$

If $\Phi(\tau)$ is the periodic solution of (19), then $\Phi(\tau+T)$ is also the periodic solution of (19), and there is the following:

$$
\Phi(\tau+T)=\mathbf{M} \Phi(\tau)
$$

where $\mathbf{M}$ is the transition matrix. Let $\tau=0$ and $\Phi(0)=I$, and there is the following:

$$
\mathbf{M}=\Phi(T)
$$

According to the Floquet theory, if the Floquet multipliers of the transition matrix $\mathbf{M}$ are all located in the unit circle, the periodic time-varying system is stable [21]. Otherwise,

- If one of the Floquet multipliers of the transition matrix $\mathbf{M}$ passes through the unit circle from the negative real axis and the rest remains in the unit circle, the perioddoubling bifurcation occurs in the periodic time-varying system;

- If one of the Floquet multipliers of the transition matrix $\mathbf{M}$ passes through the unit circle from the positive real axis and the rest remains in the unit circle, the pitch-fork bifurcation occurs in the periodic time-varying system;

- If a pair of conjugate Floquet multipliers of the transition matrix $\mathbf{M}$ goes out of the unit circle and the rest remains in the unit circle, then Hopf bifurcation occurs in the periodic time-varying system. 
Since the period of $\tau$ is $2 \pi$, that is $T=2 \pi$. Divide $T$ into equal subparts $N_{k}$, then $t_{k}=$ $T_{k} / N_{k}$. This is defined as follows:

$$
\mathbf{Y}_{k}=\frac{1}{\Delta k} \int_{t_{k-1}}^{t_{k}} \mathbf{Y}(\tau) d \tau
$$

Hence, the transition matrix $\mathbf{M}$ can be given as follows:

$$
\begin{aligned}
\mathbf{M} & =\prod_{i=N_{k}}^{1}\left[\exp \left(\mathbf{Y}_{i} \Delta_{i}\right)\right] \\
& =\exp \left(\mathbf{Y}_{N_{k}} \Delta_{N_{k}}\right) \exp \left(\mathbf{Y}_{N_{k}-1} \Delta_{N_{k}-1}\right) \ldots \exp \left(\mathbf{Y}_{1} \Delta_{1}\right) \\
& =\prod_{i=N_{k}}^{1}\left[\mathbf{I}+\sum_{j=1}^{N_{j}} \frac{\left(\mathbf{Y}_{i} \Delta_{i}\right)^{j}}{j !}\right]
\end{aligned}
$$

where

$$
\mathbf{Y}_{k}=\left[\begin{array}{ccc}
\frac{1}{\omega_{0}} \frac{1}{\Delta k} \int_{t_{k-1}}^{t_{k}} a_{11} d \tau & \frac{1}{\omega_{0}} \frac{1}{\Delta k} \int_{t_{k-1}}^{t_{k}} a_{12} d \tau & \frac{1}{\omega_{0}} \frac{1}{\Delta k} \int_{t_{k-1}}^{t_{k}} a_{13} d \tau \\
\frac{1}{\omega_{0} C} & -\frac{1}{R_{L} \omega_{0} C} & 0 \\
-\frac{k_{M} R_{v f}}{\omega_{0} R_{v i} C} & \frac{k_{M} R_{v f}}{\omega_{0} R_{v i} C R_{L}}-\frac{k_{M}}{\omega_{0} R_{v i} C_{v f}} & 0
\end{array}\right]
$$

According to (23), the Floquet multiplier of the transition matrix $\mathbf{M}$ can be calculated and used to determine the stability and bifurcation types of the periodic time-varying system. With the selected parameters in the previous section and the periodic solution obtained in (15), three Floquet multiples of the transition matrix $\mathbf{M}$ are calculated as $\lambda_{1}=2.9667 \times 10^{-4}, \lambda_{2}=2.6986 \times 10^{-21}$, and $\lambda_{3}=-2.0678 \times 10^{-20}$, respectively. Evidently, these three Floquet multiples remain in the unit circle, indicating that the voltage-controlled full-bridge inverter with a nonlinear inductor is in stable operation.

The variations of three Floquet multipliers of the transition matrix $\mathbf{M}$ with respect to the different capacitor $C_{v f}$ in the PI compensator are shown in Table 1. Additionally, two Floquet multiples $\left(\lambda_{2}\right.$ and $\left.\lambda_{3}\right)$ with different capacitors $C_{v f}$ are plotted in Figure 4. From Table 1 and Figure 4, when $C_{v f}=4 \mathrm{nF}$, three Floquet multiples (a real number and a pair of complex conjugates) of the transition matrix $\mathbf{M}$ of the voltage-controlled fullbridge inverter with a nonlinear inductor are all located in the unit circle, indicating that this system is stable. However, when $C_{v f}=3.9 \mathrm{nF}$, a pair of conjugate complex Floquet multiples of the transition matrix $\mathbf{M}$ are located outside the unit circle, indicating that the voltage-controlled full-bridge inverter with a nonlinear inductor is in unstable operation. Therefore, when the capacitor $C_{v f}$ gradually decreases from $4 \mathrm{nF}$ to $3.9 \mathrm{nF}$, there is a pair of complex conjugate Floquet multipliers penetrating from inside the unit circle and moving

\begin{tabular}{|c|c|c|c|c|}
\hline $\mathrm{C}_{\mathrm{vf}}$ & $\lambda_{1}$ & $\lambda_{2,3}$ & $\left|\lambda_{2,3}\right|$ & State \\
\hline $120.0 \mathrm{nF}$ & 0.0010 & $-5.7784 \times 10^{-20},-2.8184 \times 10^{-20}$ & Less than 0.01 & Stable \\
\hline $100.0 \mathrm{nF}$ & 0.0003 & $-4.1838 \times 10^{-21}, 7.4738 \times 10^{-21}$ & Less than 0.01 & Stable \\
\hline $80.0 \mathrm{nF}$ & $2.8828 \times 10^{-5}$ & $-3.9112 \times 10^{-22} \pm 3.3437 \times 10^{-22} i$ & Less than 0.01 & Stable \\
\hline $60.0 \mathrm{nF}$ & $7.2088 \times 10^{-7}$ & $-2.2764 \times 10^{-23}, 3.3481 \times 10^{-23}$ & Less than 0.01 & Stable \\
\hline $40.0 \mathrm{nF}$ & $3.2698 \times 10^{-10}$ & $-1.1158 \times 10^{-26} \pm 5.6199 \times 10^{-26} i$ & Less than 0.01 & Stable \\
\hline $20.0 \mathrm{nF}$ & $2.5304 \times 10^{-21}$ & $-1.5602 \times 10^{-35} \pm 7.3916 \times 10^{-34} i$ & Less than 0.01 & Stable \\
\hline $10.0 \mathrm{nF}$ & $-1.5264 \times 10^{-37}$ & $-4.8659 \times 10^{-22} \pm 1.8747 \times 10^{-22} i$ & Less than 0.01 & Stable \\
\hline $4.0 \mathrm{nF}$ & $-1.3293 \times 10^{-16}$ & $-0.9869 \pm 0.1610 i$ & 0.9999 & Stable \\
\hline $3.9 \mathrm{nF}$ & $-4.9487 \times 10^{-16}$ & $-1.7504 \pm 3.5006 i$ & 3.9139 & Unstable \\
\hline
\end{tabular}
outwards, that is, Hopf bifurcation is occurring in this system. Therefore, when $C_{v f} \geq 4 \mathrm{nF}$, the voltage-controlled full-bridge inverter with a nonlinear inductor is stable, while when $C_{v f}$ is less than or equal to $3.9 \mathrm{nF}$, the system is unstable.

Table 1. Floquet multipliers of the transition matrix $\mathbf{M}$ with respect to the different values of $C_{v f \text {. }}$ 


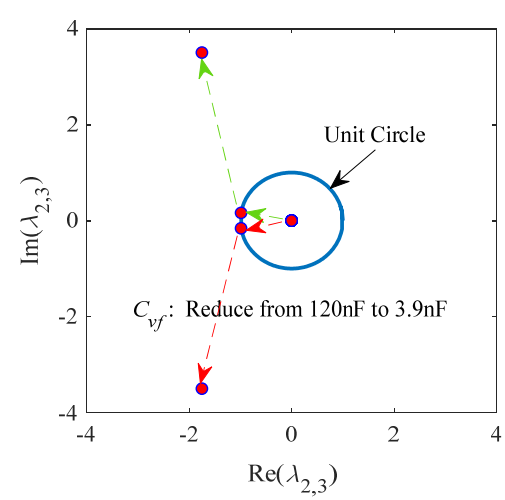

(a)

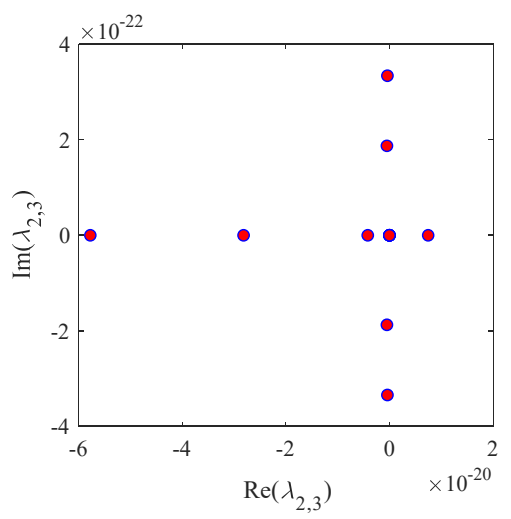

(b)

Figure 4. Changing trajectory of the eigenvalues $\lambda_{2,3}$ with respect to different $C_{v f}$ in the complex plane. (a) The eigenvalues $\lambda_{2,3}$ corresponding to Table 1 and (b) close-up view near the origin.

Therefore, when $C_{v f}=102 \mathrm{nF}$ and its value is greater than $4 \mathrm{nF}$, the voltage-controlled full-bridge inverter with a nonlinear inductor should be stable and there is no oscillation in the current $i_{N}$ and the voltage $v_{0}$, as shown in Figure 3 . However, when $C_{v f}=2 \mathrm{nF}$ and its value is less than $3.9 \mathrm{nF}$, the voltage-controlled full-bridge inverter with a nonlinear inductor is unstable. The calculated three Floquet multipliers of the corresponding transition matrix $\mathbf{M}$ under $C_{v f}=2 \mathrm{nF}$ are $\lambda_{1}=1.1686$ and $\lambda_{2,3}=3.2608 \times 10^{16} \pm 7.2821 \times 10^{15} i$, respectively. It can be seen that these three Floquet multipliers are located outside of the unit circle, which further proves that the voltage-controlled full-bridge inverter with a nonlinear inductor under $C_{v f}=2 \mathrm{nF}$ is indeed unstable.

Figure 5 shows the stability boundaries in the parameter space of the capacitor $C_{v f}$ and the capacitor $C$. One can see that with the decrease of capacitance $C_{v f}$ or the increase of capacitance $C$, the voltage-controlled full-bridge inverter with a nonlinear inductor is more likely to be unstable. Likewise, in Figure 5, for the load resistance $R_{L}=5 \Omega$, the stable region is $B_{1}+B_{2}$. However, for the load resistance $R_{L}=10 \Omega$, the stable region is $\mathrm{B}_{1}$. Hence, as the load resistance $R_{L}$ increases, the voltage-controlled full-bridge inverter with a nonlinear inductor has a smaller stable region; that is, the system is more likely to become unstable.

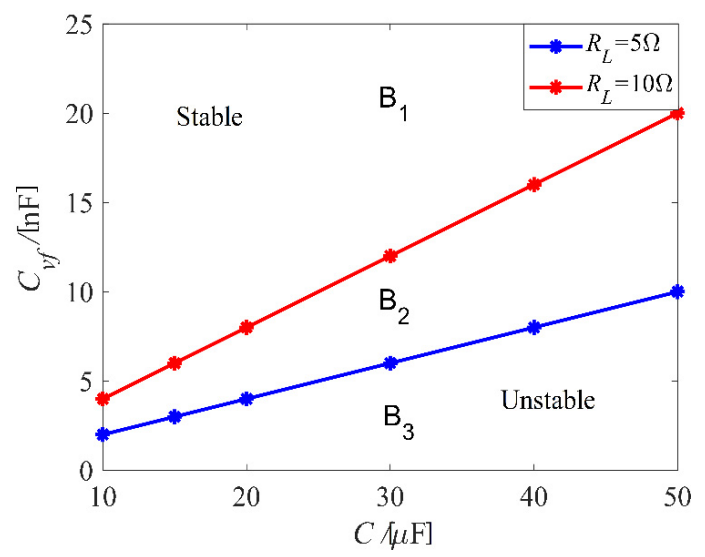

Figure 5. Stability boundaries for various capacitance $C_{v f}$ and capacitance $C$.

\section{Experimental Results}

According to the circuit structure given in Figure 2, the designed hardware experimental circuit for the voltage-controlled full-bridge inverter with a nonlinear inductor is shown in Figure 6. In the experiments, the current probe Tek A622 and the voltage differential probe P5200A were used to measure the current and the voltage in the voltage-controlled full-bridge inverter with a nonlinear inductor, and the Tek MDO-34 oscilloscope was used 
to capture the waveforms in probes. Four power switches are realized by using IRFP460, and the driver circuit used for driving these four power switches is realized by IR2130, the operational amplifier is LF356, the comparator is LM311, and the phase inverter is MAX628. In addition, the voltage sensor LV25-P $\left(i_{P}: i_{M}=1000: 2500\right)$ is used to measure the floating voltage $v_{0}$, and its magnification $k_{M}$ is calculated as follows:

$$
k_{M}=\frac{v_{M}}{v_{0}}=\frac{i_{M} R_{M}}{i_{P} R_{P}}=\frac{2500 \times 200}{1000 \times 2000}=\frac{1}{4}
$$

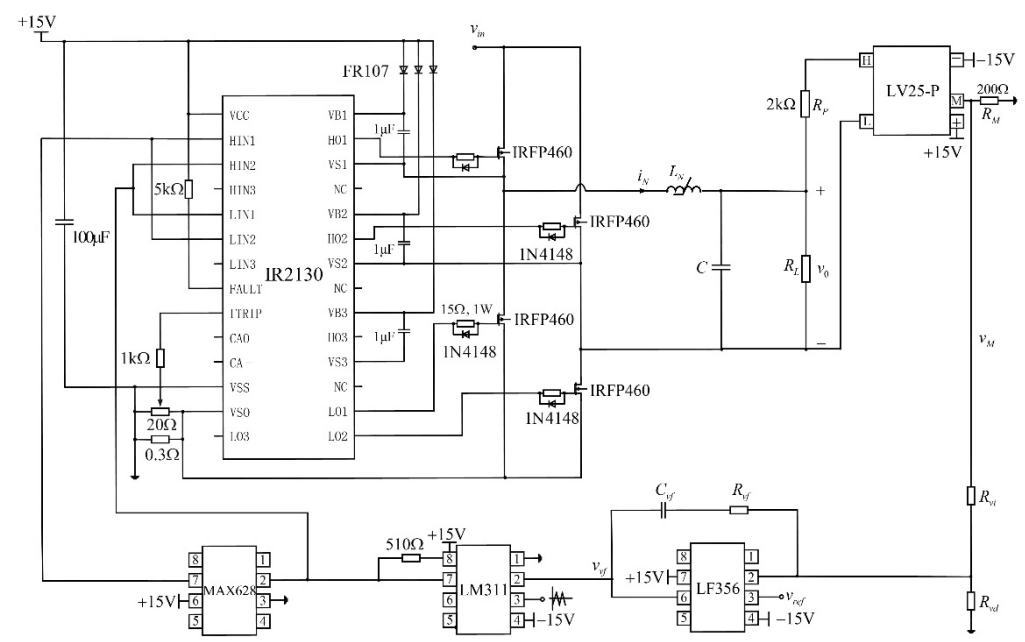

Figure 6. Experimental circuit of the voltage-controlled full-bridge inverter with a nonlinear inductor.

The other parameters remain unchanged, except when choosing a different capacitance, i.e., $C_{v f}=102 \mathrm{nF}$ and $C_{v f}=2 \mathrm{nF}$, the experimental results of the voltage-controlled full-bridge inverter with a nonlinear inductor can thus be observed. Figure 7a shows the experimental results of the current $i_{N}$ and the voltage $v_{0}$ under $C_{v f}=102 \mathrm{nF}$. Thus, when $C_{v f}=102 \mathrm{nF}$, the system is in stable operation. Moreover, the maximum value of the voltage $v_{0}$ from the experiment is $7.267 \mathrm{~V}$, and the maximum value of the current $i_{N}$ in the experiment is $1.522 \mathrm{~A}$. According to (12) and (15), the approximate calculated results for the voltage $v_{0}$ and the current $i_{N}$ are $7.482 \mathrm{~V}$ and $1.497 \mathrm{~A}$, respectively. Moreover, by comparing Figure $7 \mathrm{a}$ with Figure $3 \mathrm{a}, \mathrm{b}$, it can be seen that the experimental results are consistent with the theoretical calculation results, which verifies the correctness of the established model and the effectiveness of the approximate calculations.

In addition, with the aid of the frequency spectrum analysis of the PLECS and choosing $50 \mathrm{~Hz}$ as the fundamental frequency, the experimental curves of the current $i_{N}$ and the voltage $v_{0}$ can be obtained, as shown in Figure $7 \mathrm{c}, \mathrm{d}$, respectively. One can see that in the range of $0 \sim 1 \mathrm{kHz}$, the harmonics of both the current $i_{N}$ and the voltage $v_{0}$ have smaller amplitudes than the fundamental wave. In the range of $1-2 \mathrm{kHz}$, the amplitude of each harmonic can be approximately zero.

Figure 8a illustrates the experimental curves of the current $i_{N}$ and the voltage $v_{0}$ under $C_{v f}=2 \mathrm{nF}$, while Figure $8 \mathrm{~b}$ is the close-up view of Figure 8a. Figure 8c,d are the harmonic spectrums of the current $i_{N}$ and the voltage $v_{0}$, respectively. It can be seen that when $C_{v f}=2 \mathrm{nF}$, the voltage-controlled full-bridge inverter with a nonlinear inductor is in unstable operation and there is oscillation; the oscillation frequency is between the power frequency of $50 \mathrm{~Hz}$ and the switching frequency of $25 \mathrm{kHz}$. This oscillation phenomenon can be called medium-frequency oscillation. In the region of the oscillation frequency, both the current $i_{N}$ and the voltage $v_{0}$ have larger harmonics. Therefore, the occurrence of medium-frequency oscillation will increase the difficulty of the system filter design; likewise, will increase the voltage stress and the current stress of the power switches in the system. 


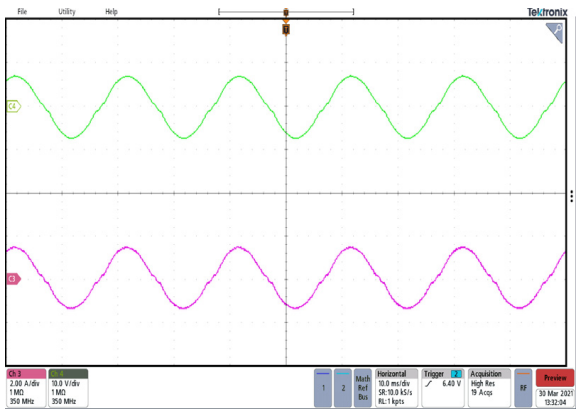

(a)

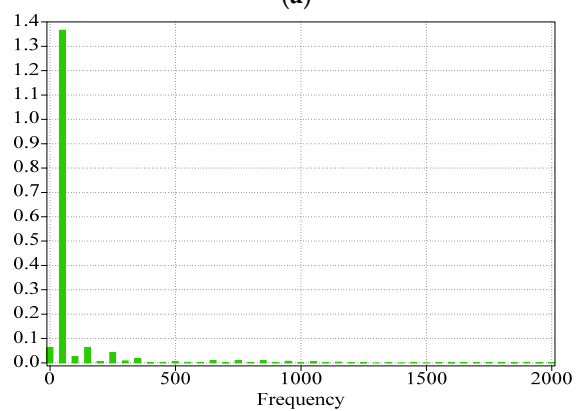

(c)

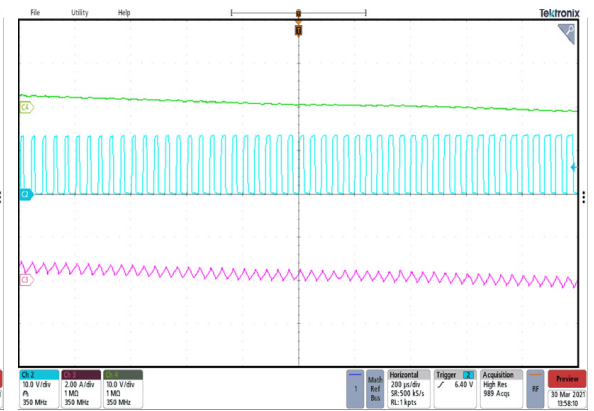

(b)

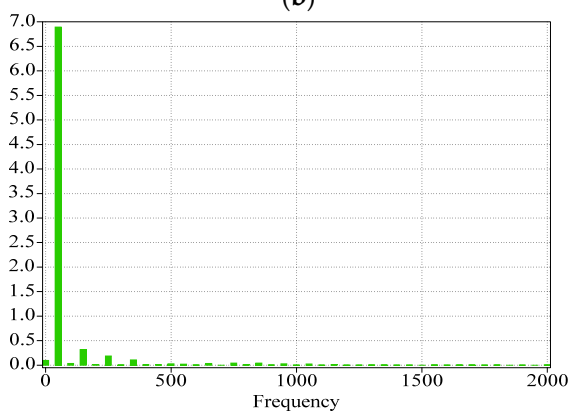

(d)

Figure 7. Experiments for $C_{v f}=102 \mathrm{nF}$. (a) $v_{0}(\mathrm{Ch} 4: 10 \mathrm{~V} / \mathrm{div})$ and $i_{N}(\mathrm{Ch} 3: 2 \mathrm{~A} / \mathrm{div})$, time: $10 \mathrm{~ms} / \mathrm{div}$. (b) Close-up view, $v_{0}(\mathrm{Ch} 4: 10 \mathrm{~V} / \mathrm{div}), i_{N}(\mathrm{Ch} 3: 2 \mathrm{~A} / \mathrm{div})$, and $v_{d}(\mathrm{Ch} 2: 10 \mathrm{~V} / \mathrm{div})$, time: $200 \mu \mathrm{s} / \mathrm{div}$. (c) Harmonic spectrum of $i_{N}$ [axis X: Hz, axis Y: A]. (d) Harmonic spectrum of $v_{0}$ [axis X: Hz, axis Y: V].

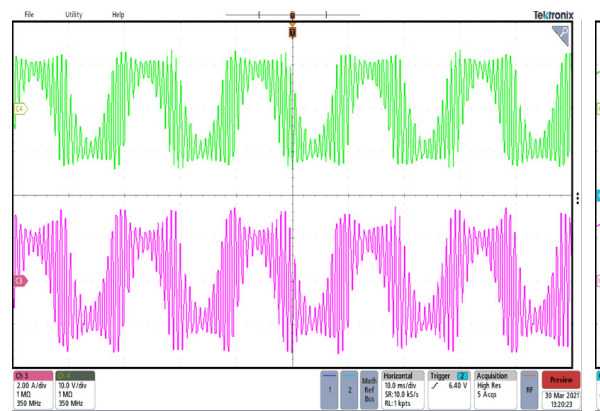

(a)

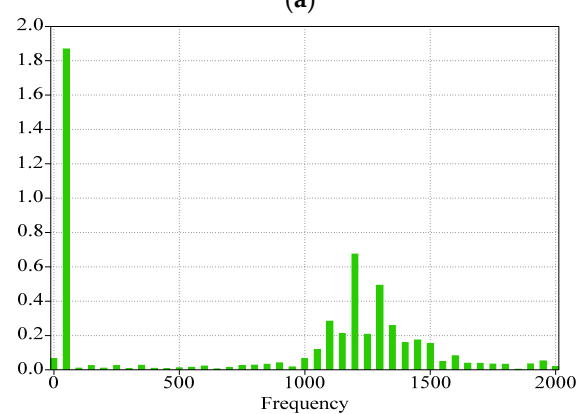

(c)

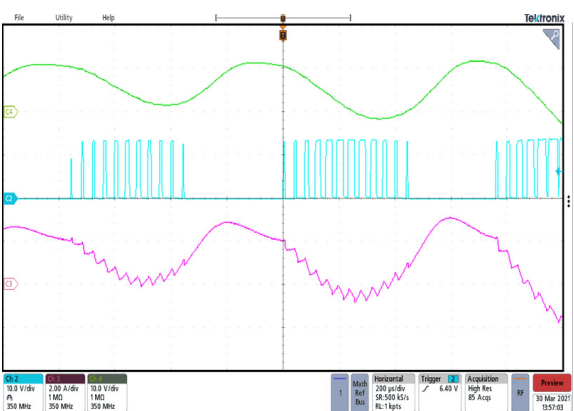

(b)

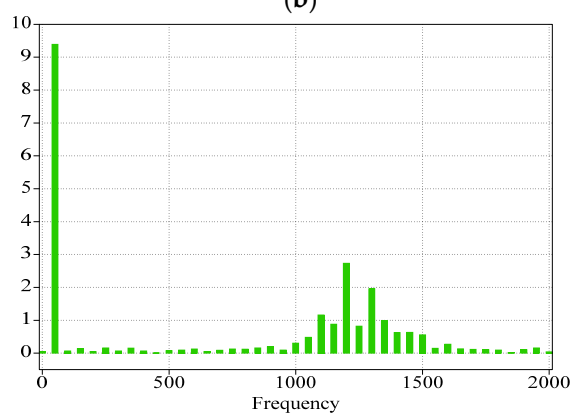

(d)

Figure 8. Experiments for $C_{v f}=2 \mathrm{nF}$. (a) $v_{0}(\mathrm{Ch} 4: 10 \mathrm{~V} / \mathrm{div})$ and $i_{N}(\mathrm{Ch} 3: 2 \mathrm{~A} / \mathrm{div})$, time: $10 \mathrm{~ms} / \mathrm{div}$. (b) Close-up view, $v_{0}(\mathrm{Ch} 4: 10 \mathrm{~V} / \mathrm{div}), i_{N}(\mathrm{Ch} 3: 2 \mathrm{~A} / \mathrm{div})$, and $v_{d}(\mathrm{Ch} 2: 10 \mathrm{~V} / \mathrm{div})$, time: $200 \mu \mathrm{s} / \mathrm{div}$. (c Harmonic spectrum of $i_{N}$ [axis X: Hz, axis Y: A]. (d) Harmonic spectrum of $v_{0}$ [axis X: Hz, axis Y: V].

\section{Conclusions}

In this paper, the results from theoretical analysis, approximate calculation, stability determination, and circuit experiment show that in the voltage-controlled full-bridge inverter with a nonlinear inductor, the medium-frequency oscillation whose oscillation 
frequency is between the power frequency and the switching frequency causes the occurrence of Hopf bifurcation, making the filter design for the system more difficult since this oscillation should be filtered. Hence, the medium-frequency oscillation occurring in the voltage-controlled full-bridge inverter with a nonlinear inductor should be avoided in practical engineering.

Author Contributions: F.W. conceived, validated, and wrote the manuscript. H.Z., J.Z. and H.C. participated in the research plan development and revised the manuscript. All authors have read and agreed to the published version of the manuscript.

Funding: This work was supported in part by the National Natural Science Foundation of China under Grant 51377124.

Conflicts of Interest: The authors declare that there are no conflicts of interest regarding the publication of this paper.

\section{References}

1. Luo, F.L.; Hong, Y. Advanced DC/AC Inverters: Applications in Renewable Energy; CRC Press Taylor and Francis Group: Boca Raton, FL, USA, 2013; pp. 33-135.

2. Shen, J.L.; Chen, W. Nonlinear analysis and improvement of output filtering inductance for single-phase photovoltaic inverter. J. Magn. Mater. Dev. 2020, 51, 37-42.

3. Yang, C.Y.; Chen, W. Analysis of effect of ladder gap on nonlinear characteristics of inductors. J. Power Supply 2012, 4, 39-43.

4. Wang, L.L.; Qiu, Y.J.; Wang, H.L.; Hu, Z.Y.; Liu, Y.F. A new model for designing multiwindow multipermeability nonlinear LTCC inductors. IEEE Trans. Ind. Appl. 2015, 51, 4677-4686. [CrossRef]

5. Liu, Y.T.; Zhang, D.M. Effect of nonlinear inductor behavior on the performance of interleaved power factor correction. IEEE Trans. Magn. 2012, 48, 4143-4147. [CrossRef]

6. Alexander, S.; Christof, G. Efficient nonlinear inductors for PV inverters and active PFC. In Proceedings of the 7th International Conference on Integrated Power Electronics Systems, Nuremberg, Germany, 6-8 March 2012; pp. 1-4.

7. Liu, Y.T.; Jiang, S.Q.; Wang, H.Z.; Wang, G.B.; Peng, J.C.; Liu, Y. LCL filter design with the inductor nonlinear behavior consideration in the three phase grid-connected inverter. In Proceedings of the 2018 IEEE International Magnetics Conference, Singapore, 23-27 April 2018; p. 1800026.

8. Mastromauro, R.A.; Liserre, M.; Dell'Aquila, A. Study of the effects of inductor nonlinear behavior on the performance of current controllers for single-phase PV grid converters. IEEE Trans. Ind. Electron. 2008, 55, 2043-2052. [CrossRef]

9. Li, H.; Zhou, Y.F.; Ling, Q.Q. Modeling and dynamics of boost converter based on nonlinear inductor. Electr. Meas. Instrum. 2019, $56,39-44$.

10. Sun, L.X.; Zhou, Z.Y.; Wen, Z.G.; Wang, Q.Y. Adaptive carrier amplitude modulation control of bifurcation and chaos in SPWM H-bridge converter. Electr. Meas. Instrum. 2020, 57, 101-108.

11. Gong, R.X.; Yin, Z.H. A chaos control method of single-phase H-bridge photovoltaic inverter. Acta Phys. Sin. 2021, 70, 020501.

12. Yang, L.H.; Yang, L.; Yang, F.; Ma, X.K. Slow-scale and fast-scale instabilities in parallel-connected single-phase H-bridge inverters: A design-oriented study. Int. J. Bifurc. Chaos 2020, 30, 2050005. [CrossRef]

13. Liu, H.C.; Su, Z.X. Study on nonlinear phenomena in dual buck full-bridge inverter. Acta Phys. Sin. 2014, 63, 010505.

14. Zhang, J.K.; Wu, X.J.; Xing, L.S.; Zhang, C.; Iu, H.; Fernando, T. Bifurcation analysis of five-level cascaded H-bridge inverter using proportional-resonant plus time-delayed feedback. Int. J. Bifurc. Chaos 2016, 26, 1630031. [CrossRef]

15. Lenz, E.; Pagano, D.J.; Ruseler, A.; Heldwein, M.L. Two-parameter stability analysis of resistive droop control applied to parallel-connected voltage-source inverters. IEEE J. Emerg. Sel. Top. Power Electron. 2020, 8, 3318-3332. [CrossRef]

16. Chen, Y.; Zheng, Y.; Jiang, L. Nonlinear dynamic behavior of high-frequency isolation quasi-Z-source photovoltaic inverter. IEICE Electron. Express 2018, 15, 1-12. [CrossRef]

17. Liao, Z.X.; Luo, X.S.; Huang, G.X. Numerical modeling and research on nonlinear dynamic behaviors of two-stage photovoltaic grid-connected inverter. Acta Phys. Sin. 2015, 64, 130503.

18. Dai, Y.Z.; Zhao, P.C.; Ren, H.J.; Luo, R.Z. Rorder-collision bifurcation and stability domain of non-isolated photovoltaic gridconnected inverter with H6-type. Acta Energ. Solaris Sin. 2019, 40, 126-133.

19. Asadi, F.; Eguchi, K. Simulation of Power Electronics Converters Using PLECS; Elsevier Academic Press: Amsterdam, The Netherlands, 2020; pp. 399-419.

20. Middlebrook, R.D.; Cuk, S. A general unified approach to modelling switching-converter power stages. Int. J. Electron. 1997, 42, 521-550. [CrossRef]

21. Wang, F.Q.; Zhang, H.; Ma, X.K. Period-doubling bifurcation in two-stage power factor correction converters using the method of incremental harmonic balance and Floquet theory. Chin. Phys. B 2012, 21, 020505. [CrossRef] 